\title{
Rafael Yuste, neurobiólogo profesor en la Universidad de Columbia, sobre neuroderechos y el planteamiento sobre su reconocimiento y protección como derechos humanos
}

\author{
Claudia Iriarte Rivas (D) y Alejandra Olivares Muñoz iD \\ Universidad de Chile
}

Estamos iniciando un proceso constituyente que es bastante inédito para la historia chilena. Se ha elegido y constituido una comisión constitucional, que, si bien no es exactamente una asamblea constituyente, es una nueva institucionalidad que permite un debate ciudadano, conducente a la elaboración de un nuevo texto constitucional. Parte de estos debates abordarán nuevos temas en materia de derechos humanos, como es el referido al de los neuroderechos. Existe actualmente un proyecto de ley que se debate en el Congreso Nacional — que incluye una reforma constitucional-, el cual queda en suspenso ante el proceso constituyente que estamos inaugurando.

En este contexto, es para nosotros del mayor interés recoger la opinión de quienes abordan y han elaborado propuestas relacionadas con los planteamientos sobre neuroderechos y la necesidad de garantizarlos en el marco de un sistema jurídico de derechos humanos, como un aporte para el debate sobre nueva constitución, derechos humanos y sistema de garantías de estos.

Es en este marco en el cual organizamos esta entrevista al profesor Rafael Yuste, neurobiólogo, profesor de Ciencias Biológicas en la Universidad de Columbia, Estados Unidos, e impulsor de una propuesta que plantea la necesidad de establecer y proteger los neuroderechos, tanto en el sistema internacional de los derechos humanos como en los ordenamientos jurídicos constitucionales y legales de los países.

Lo primero: ¿qué son las neurotecnologías y cuáles son los principales riesgos que estas conllevan?

Las neurotecnologías son métodos que pueden ser ópticos, eléctricos, de nanociencia, químicos o magnéticos para hacer dos cosas: para registrar la actividad del cerebro y 
para alterar la actividad del cerebro. Las neurotecnologías se están desarrollando actualmente en Estados Unidos y en muchos otros sitios del mundo, y son importantes porque el cerebro no es un órgano cualquiera de cuerpo, sino que es el órgano que genera la mente humana, la actividad cerebral genera todas las propiedades cognitivas y mentales de los seres humanos. Por eso, las neurotecnologías pueden acceder a la actividad mental y cambiarla, y esto no es ciencia ficción, sino algo que hacemos rutinariamente en animales, en el laboratorio, y se puede empezar a vislumbrar cómo se puede utilizar esto en humanos también.

¿Cuál es el vínculo desde la idea de las neurotecnologías y los riesgos que estas conllevan, ahora expresada en la idea de neuroderechos, y por qué es necesaria la garantía y protección de estos neuroderechos?

Por el potencial que tienen las neurotecnologías de poder acceder a la información cerebral y cambiarla, tienen la posibilidad de cambiar la actividad mental, y la actividad mental es la esencia del ser humano. Estoy hablando de todo lo que somos mentalmente, nuestras percepciones, nuestras ideas, nuestra imaginación, nuestras memorias, nuestras emociones, nuestros planes, nuestras decisiones, todo esto surge de la actividad cerebral. Nosotros pensamos que es necesario proteger la actividad cerebral de potenciales abusos con la neurotecnología. Y como la actividad cerebral genera la mente humana, pensamos que es un problema de derechos humanos; que tenemos que proteger la actividad cerebral como un derecho humano básico, porque si no la mente puede ser objeto de manipulaciones y desciframientos indebidos.

En Chile ha habido un debate a propósito de esta temática y hay quienes sostienen que no es necesario avanzar hacia la protección de estos como nuevos derechos, que basta con reconceptualizar los que ya existen -como son, por ejemplo, el derecho a la integridad física y síquica, el derecho a la libertad y seguridad individual y el respeto $y$ protección a la vida privada y los datos personales-, pero, en su propuesta, al parecer esto no es suficiente. ¿Usted cree que en realidad necesitamos avanzar hacia la configuración de nuevos derechos humanos, que incluso se requiera modificar los principales instrumentos internacionales en materia de derechos humanos?

Yo pienso que sí. De hecho, hablo en nombre de un grupo amplio de expertos que somos el Grupo Morningside, que nos reunimos en la Universidad de Columbia en 2017 y propusimos el concepto de neuroderechos. Nuestra posición es que los derechos humanos existentes, como el derecho humano a la dignidad de la persona, no son suficientes, porque la Declaración Universal que se formuló en 1948 no estaba pensada para el mundo en el que vivimos ahora, en que las tecnologías han avanzado muchísimo. Por ejemplo, el artículo 28 de la Declaración Universal de Derechos Humanos, referido al derecho humano a la dignidad, se puede interpretar de muchas 
maneras. Yo creo que si tenemos a diez personas en una habitación y le preguntas a cada una qué entiende por dignidad personal, todas tendrán una respuesta distinta.

Por ello, a los 29 derechos humanos existentes, pensamos que hay que añadir nuevos derechos humanos que definan específicamente y de una manera clara la protección que es necesaria con respecto a este mundo futuro.

Estamos proponiendo el derecho, por ejemplo, a la identidad personal. Esta es una cosa que nosotros no creemos que esté recogida en la Declaración Universal de Derechos Humanos, porque nadie se hubiese imaginado en 1948 que te pueden cambiar la identidad, el yo, que quien es la persona que tú eres puede ser cambiado. Esto es una cosa que puede ocurrir con neurotecnología y, de hecho, está ocurriendo, con pacientes que reciben estimulación cerebral profunda. Este es el primer neuroderecho que nosotros añadiríamos a la Declaración Universal: el derecho a la identidad personal, al yo. De hecho, en mi opinión personal, ese tendría que ser el primer derecho humano, porque si no tienes el derecho a tu propia identidad personal, para qué sirven el resto de los derechos humanos, a quién se refieren el resto de los derechos humanos.

El segundo neuroderecho que pensamos nosotros que no está absolutamente bien recogido es el derecho a la agencia, a la libre decisión, al libre albedrío. Es una cosa que tampoco se hubiese pensado que fuera necesario en el año 1948, porque quién se hubiese imaginado que te podrían cambiar la actividad cerebral para que decidas una cosa en vez de otra. Esto, que parece ciencia ficción, lo hacemos ya con ratones. Lo que se puede hacer hoy con ratones se podrá hacer con humanos mañana y tenemos que estar preparados, definiendo un derecho universal al libre albedrío, para que las decisiones de las personas no sean interferidas desde el exterior con técnicas de neurotecnologías.

Luego, tenemos también el tercer neuroderecho que proponemos, que es el derecho a la privacidad mental. Esto va mucho más allá en nuestra opinión de los derechos a la privacidad de los datos que existen actualmente en el mundo, que en algunas partes son más fuertes que otros, porque estos se refieren a datos personales que puedes tener almacenados en tu teléfono móvil, en tu ordenador, o a datos sobre cómo te mueves en la ciudad, y es importante que esto se proteja, pero creemos que se queda corto a la hora de proteger los datos cerebrales. ¿Por qué decimos esto? Porque los datos cerebrales representan no lo que hace tu cuerpo, dónde estás localizado o lo que escribes, sino lo que piensas. Entonces, ahí hay un salto de nivel de privacidad en el que entramos en el corazón de la privacidad personal, y desde nuestro punto de vista ya no hay más privacidad, es la privacidad máxima, lo que piensa la persona, el pensamiento, el contenido de la mente de la persona. Nosotros pensamos que esto debe ser definido específicamente y tratado de una manera jurídica distinta a la privacidad normal de los datos a los cuales estamos acostumbrados.

Por último, nosotros proponemos dos nuevos derechos humanos relacionados 
con la neurotecnología. Uno que garantice el acceso equitativo a las tecnologías de neuroaumentación. Estamos hablando de tecnologías que todavía no existen, pero que se están empezando a desarrollar para aumentar cognitivamente al ser humano. Esto va a crear una humanidad de dos velocidades, con seres humanos que estén aumentados cognitivamente y seres humanos que no lo estén. Por eso nosotros queremos asegurarnos de que antes de que estas tecnologías se esparzan por la población, estén reguladas bajo el principio universal de justicia, para no romper el acceso equitativo a estas tecnologías que pueden cambiar la esencia del ser humano. Eso también te da una idea de por qué pensamos que es un problema de derechos humanos, porque estamos hablando de la esencia de lo que significa ser una persona.

Para terminar, el último derecho humano que estamos proponiendo es el derecho a ser protegido de los sesgos de los algoritmos que se utilizan y se van a utilizar en neurología. Estos algoritmos, desafortunadamente, muchas veces tienen un sesgo que, con la neurotecnología implantada directamente en nuestro cerebro, serían incorporados en nuestra mente como algo propio, como algo que pensamos nosotros, no como algo que venga desde fuera. Esto es algo de lo que tenemos que estar protegidos con ojo avizor hacia el futuro y evitarlo antes de que ocurra.

He dado una respuesta larga, pero no sé si he cubierto lo que quería usted saber.

Sí, absolutamente. Solo para cerrar un poco la idea, porque nos parece muy interesante esto que ustedes están planteando respecto al instrumental internacional de derechos humanos, ¿piensan que basta con el reconocimiento de los derechos o habría que avanzar en sistemas de garantías, que en derecho son normas que acompañan estos derechos, pero que establecen garantías específicas de no intervención o de protección, o ustedes creen que con reconocimiento es ya un avance sustancial?

Nosotros pensamos que primero es necesario poner las bases de los principios éticos y jurídicos a través de los derechos humanos y después desarrollarlos y aterrizarlos en las normativas específicas de cada país a través de una legislación que garantice su respeto, su ejecución y su protección, porque si no serían papel mojado. En el caso de Chile, por ejemplo, el proyecto de reforma del artículo 19 de la Constitución, que ha sido aprobado por el Senado y se está discutiendo en la Cámara, y el proyecto de ley de neuroderechos, de neuroprotección, que está discutiendo el Senado actualmente, son dos medidas cuyo espíritu es no solo proponer y describir los derechos, sino garantizarlos a través de la aplicación de legislación existente en Chile y, en concreto, aplicar el Código Sanitario a las neurotecnologías, de manera que se incorporen dentro del tipo de tecnologías médicas que ya tienen un alto grado de protección legal.

No sé si me explico, la idea es ampliar la definición de lo que representa la medicina desde el punto de vista legal, para que se incorporen las neurotecnologías como dispositivos médicos, aunque sean dispositivos que no requieran cirugía, aunque 
puedas comprar estos dispositivos en una tienda como si fuese un teléfono móvil. A pesar de todo, la idea sería que estuviesen regulados como dispositivos médicos; en este caso, se garantizaría la aplicación de estas normas de derechos en estos dispositivos y en esta tecnología cuando llegue al consumidor, cuando llegue a la ciudadanía.

Considerando lo que usted nos señaló en cuanto a los riesgos que conllevaban estas neurotecnologías y a los avances científicos que ha habido en materia de neurotecnología, queríamos preguntarle: ¿cuáles son las directrices éticas, desde la perspectiva de los neuroderechos, que usted considera imprescindibles en la práctica de la neurociencia y en las investigaciones tecnológicas que trabajan con el cerebro humano?

Interpretamos los derechos humanos como una base ética sobre la cual se puede construir y desarrollar la neurotecnología del futuro, y como yo comentaba, el otro aspecto de nuestras propuestas tiene que ver con lo que se podría llamar el modelo médico, que, como estaba describiendo antes, consiste en aplicar el Código Sanitario, los principios médicos, para dirimir y regular esta tecnología, en vez de reinventar la rueda y hacerlo todo de nuevo e inventar una serie de leyes nuevas para esta tecnología que puede tener tanta importancia y puede ser rompedora para bien y para mal en la sociedad.

Si tú escarbas un poco en el centro de la medicina, se encuentra el Código Ético, desde hace un tiempo, desde hace más de 2.000 años; el juramento hipocrático es asumido por todos los practicantes de la medicina en todas las historias, en todas las culturas y en todo el mundo. El juramento hipocrático ha sido codificado recientemente en el siglo XX de una manera más moderna en los principios del informe Belmont. Los tres principios de Belmont son muy simples: el principio de beneficencia, actuar para hacer bien al prójimo; el principio de justicia, tratar a todos los pacientes de la misma manera sin hacer distinciones; y, por último, el principio médico de digni$\mathrm{dad}$, que significa en este caso tratar al paciente, a las personas, como seres humanos y no como objetos.

Por ello, en respuesta a tu pregunta, si se incorpora, si se aplica el modelo médico a la neurotecnología, estos tres principios éticos pasarían a ser una especie de código tecnocrático, juramento tecnocrático en vez de juramento hipocrático de los médicos, y se aplicaría, sería asumido como propio por los practicantes de la neurotecnología, tanto la gente que diseña estos dispositivos como la gente que los aplica y los lleva a la población. Estamos hablando de una situación en la cual compañías tecnológicas que, por ejemplo, venden ahora teléfonos móviles, o aplicaciones como Facebook, si se incorporan al desarrollo y aplicación de estas tecnologías, tendrían que tener también una especie de juramento tecnocrático, para asegurarse de que tengan unos principios éticos en la base de todo. 
Ahora queremos preguntarle un aspecto que consideramos que es más un desafío que presentarían las neurotecnologías y su regulación, considerando que las generaciones actuales y las futuras van a estar inmersas en un mundo en que la alta presencia de esta tecnología resultará natural. ¿Usted considera que la regulación de los neuroderechos va a tener un sentido ético para estas nuevas generaciones?

Las nuevas generaciones van a ser afectadas tanto por las neurotecnologías como por otras tecnologías digitales: la inteligencia artificial, por ejemplo, y yo estoy convencido de que ya han empezado a cambiar las reglas del juego del mundo. De hecho, parte de los problemas que tenemos las sociedades en la actualidad, los problemas políticos y sociales, tienen que ver con el carácter disruptivo de la inteligencia artificial y de las tecnologías digitales.

Creo que la aparición de las neurotecnologías va a ser incluso más potente que todas las tecnologías artificiales que tenemos hasta ahora, como las redes sociales. ¿Por qué digo esto? Porque va a afectar a la esencia del humano, que es la mente humana, por lo que esto nos fuerza a plantearnos el problema de la regulación futura de todas las tecnologías digitales como uno de derechos humanos. Ahí es cuando los derechos pueden tener una importancia seminal o capital en la discusión de la regulación de las nuevas tecnologías digitales en el nuevo proceso constituyente en Chile, porque es un poco imaginarse que los neuroderechos pueden servir como punta de lanza para todos los derechos digitales, no solamente que tengan que ver con la neurotecnología, ya que, una vez que abres el tema, planteas el problema de las neurotecnologías en la arena de los derechos humanos, ya que pueden englobar no solo las neurotecnologías, sino todas las tecnologías digitales.

Yo creo que esto sería lo mejor que podríamos hacer para proteger a las generaciones futuras, a los niños y adolescentes, para que en vez del descontrol que existe en la actualidad - en que las tecnologías digitales se están utilizando de una manera masiva y sin principios éticos detrás-, se enfoque todo el tema desde el punto de vista de los derechos humanos. Podríamos decir primero qué tipo de ser humanos queremos ser y que sean las nuevas generaciones, y con estos principios éticos ya armados, podríamos incorporar todas las tecnologías digitales de una manera que coincida con el ser humano que queremos ser, en vez de dejarnos llevar por la tecnología sin ningún principio ético detrás, con principios comerciales o incluso con cuestiones de azar que decidan el futuro de la humanidad.

Con certeza, creo que este problema va a tener que ser debatido en la convención constitucional, considerando que claramente esta constitución va a regir para las futuras generaciones, quienes son aquellas que ya están inmersas y naturalizan la presencia de la alta tecnología a través de las redes sociales, como Facebook o Twitter, y los dispositivos móviles. 
Quisiera añadir a esta última pregunta la siguiente reflexión: actualmente $-\mathrm{y}$ esto es algo que hemos visto incluso con el covid-19- pasamos gran parte de nuestra vida en el mundo digital, y esto va a más, no a menos. Por otro lado, tenemos constituciones que están hechas para otro mundo, el real y físico.

Por esa razón, la oportunidad del proceso constituyente en Chile es única, para poder replantearse una constitución de un país para un mundo digital, en que por supuesto se sigue regulando el mundo físico, pero, a la vez, en que gran parte de la actividad humana se da en el mundo digital. Esto hace del proceso una oportunidad, y la irrupción de la neurotecnologia te fuerza a hacerlo. Nosotros pensamos que no hay otra manera de integrarla en la sociedad que no sea replanteándola desde el punto de vista de los derechos humanos. Tenemos que definir primero en este mundo digital qué tipo de ser humano queremos ser. Esto se tiene que ampliar, no solo a la neurotecnología, sino a todas las tecnologías digitales.

Yo creo que ustedes tienen una gran oportunidad en el marco del proceso constituyente de contar con un libro en blanco, una nueva constitución hacia un mundo futuro. Esto sería una oportunidad histórica para que Chile lidere a otros países en la definición de las reglas del juego del futuro, del mundo digital. En otros países hay parches legales para intentar tapar los agujeros que surgen de la aplicación masiva de estas tecnologías digitales en una sociedad que no está preparada para ello. Es la solución que tenemos en las sociedades occidentales que protegen, por ejemplo, la propiedad de los datos, el uso del agente artificial de reconocimiento facial, que no es una situación ideal para poner parches. Es llegar tarde al problema y tratar de tapar el agujero. Mientras que, si replanteas el problema desde el comienzo como uno de derechos humanos, no solo para la neurotecnologia, sino para todas las tecnologías digitales, tendríamos bien armado un frente jurídico y ético mucho más fuerte y potente.

Bien, profesor, esa es la última pregunta que teníamos, agradecemos mucho su presencia aquí, considero que esta entrevista va a ser fructífera para el futuro de Chile, para la discusión que estamos teniendo a nivel constitucional, así que agradecerle nuevamente que haya concedido esta entrevista al Centro de Derechos Humanos.

Agradecemos su entrevista, profesor.

\section{Sobre las autoras}

Claudia Iriarte Rivas es abogada. Doctora en Derecho, investigadora del Centro de Derechos Humanos de la Facultad de Derecho de la Universidad de Chile. Actualmente se desempeña como editora del Anuario de Derechos Humanos. Su correo electrónico es ciriarter@derecho.uchile.cl. (D) https://orcid.org/00oo-0003-0449-926X. 
Alejandra Olivares Muñoz es egresada de Derecho de la Universidad de Chile y ayudante ad honorem del Centro de Derechos Humanos de la misma casa de estudios. Participó como moderadora en la Escuela de Formación de Formadores en Derechos Humanos «La centralidad de los derechos humanos en el debate constitucional», organizada por el mismo centro. Fue ayudante de las cátedras de Derecho Constitucional y de Orden Público y Recursos de Protección y Amparo Económico. En 2019, cursó un intercambio académico en la Universidad de São Paulo, Brasil. Su correo electrónico es alejandra.olivaresmunoz@gmail.com. (D) https://orcid. org/0000-0001-8326-130X. 
El Anuario de Derechos Humanos es una publicación semestral de referencia y consulta en materia de derechos humanos y campos afines. Busca ser un espacio de discusión de los temas centrales en el ámbito nacional e internacional sobre derechos humanos. Es publicado desde 2005 por el Centro de Derechos Humanos de la Facultad de Derecho de la Universidad de Chile.

\author{
EDITORA \\ Claudia Iriarte Rivas \\ ciriarter@derecho.uchile.cl \\ SITIO WEB \\ anuariocdh.uchile.cl \\ CORREO ELECTRÓNICO \\ anuario-cdh@derecho.uchile.cl \\ LICENCIA DE ESTE ARTÍCULO \\ Creative Commons Atribución Compartir Igual 4.o Internacional
}

\author{
\% \\ La edición de textos, el diseño editorial \\ y la conversión a formatos electrónicos de este artículo \\ estuvieron a cargo de Tipográfica \\ (www.tipografica.io)
}

